\title{
High b value DWI in evaluation of the hyperacute cerebral ischemia at 3T: A comparative study in an embolic canine stroke model
}

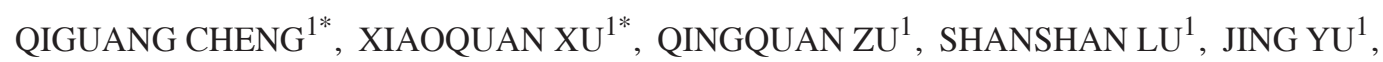 \\ XINGLONG LIU ${ }^{1}$, BIN WANG $^{1}$, HAIBIN SHI $^{1}$, GAOJUN TENG $^{2}$ and SHENG LIU ${ }^{1}$ \\ ${ }^{1}$ Department of Radiology, The First Affiliated Hospital of Nanjing Medical University; \\ ${ }^{2}$ Department of Radiology, Zhongda Hospital, Medical School of Southeast University, Nanjing, Jiangsu 210029, P.R. China
}

Received April 4, 2015; Accepted May 5, 2016

DOI: $10.3892 /$ etm.2016.3403

\begin{abstract}
Previous studies have indicated that the temporal change of relative diffusion weighted imaging (rDWI) signal intensity may help to determine the onset time of a stroke. Furthermore, several studies have indicated that high $b$ value DWI offered improved detection rates for hyper-acute ischemic lesions compared with standard b value DWI. However, the temporal changes of the rDWI on high $b$ value DWI remain unclear. Therefore, based on our embolic canine stroke model, we evaluated the temporal evolution of rDWI on high $b$ value DWI, and further compared its diagnostic value in predicting the onset time of ischemic stroke with rDWI on standard b value DWI. Twelve canine MCAO models were established, and DWI was performed at 1, 2, 3, 4, 5 and $6 \mathrm{~h}$ after MCAO, with $3 \mathrm{~b}$ values of 1,000, 2,000 and 3,000. High b value DWI detected all ischemic lesions after $1 \mathrm{~h}$, while standard $\mathrm{b}$ value did not detect the ischemic lesions in one dog at $1 \mathrm{~h}$. With all three of the tested $b$ values, rDWIs increased continuously within $6 \mathrm{~h}$, while relative apparent diffusion coefficient (rADC) values rapidly decreased in $1 \mathrm{~h}$, then became relatively stable. The area under the curve values for rDWI with $b$ value of 1,000, 2,000 and 3,000, in predicting ischemic lesions within $3 \mathrm{~h}$ were $0.897,0.929$ and 0.938 , while for rADC were 0.645 ,
\end{abstract}

Correspondence to: Dr Sheng Liu, Department of Radiology, The First Affiliated Hospital of Nanjing Medical University, 300 Guangzhou Road, Nanjing, Jiangsu 210029, P.R. China

E-mail: liusheng@njmu.edu.cn

*Contributed equally

Abbreviations: rSI, relative signal intensity; rDWI, relative diffusion weighted image; rADC, relative apparent diffusion coefficient; ECA, external cerebral artery; ICA, internal cerebral artery; MCA, middle cerebral artery; $\mathrm{MCAO}$, middle cerebral artery occlusion

Key words: cerebral ischemia, onset time, relative signal intensity, diffusion weighted imaging, high $b$ value
0.583 and 0.599 , respectively. Therefore, the results indicated that the rDWI was helpful in aging hyper-acute ischemic stroke, while rADC appeared not to be. High b value DWI had a higher detection rate for ischemic lesions and better predictive efficacy in determining the onset time of hyper-acute stroke.

\section{Introduction}

Diffusion-weighted imaging (DWI) is a non-invasive imaging technique which can provide quantitative information about Brownian motion in normal or abnormal tissue (1). DWI enables the measurement of the apparent diffusion coefficient (ADC), and has been established as an essential magnetic resonance (MR) sequence for stroke imaging $(2,3)$. DWI has been shown to be highly sensitive for detecting hyper-acute cerebral ischemia, as early as $30 \mathrm{~min}$ after arterial occlusion $(4,5)$. Furthermore, a number of studies have indicated that the relative signal intensity (rSI) of DWI may provide useful information about the time course of ischemia events, help to determine the onset time of ischemic lesions and select optimal management for patients with acute ischemic stroke (6-9).

Recently, with the improvement in the MR gradient technology and field strength, increased diffusion sensitivity without significant loss of signal to noise ratio (SNR) is now possible (10). As a result, high b value DWI has been applied in stroke related studies. For example, previous studies indicated that high $b$ value DWI could detect more ischemia lesions earlier in the same period (11-14). However, the temporal changes of the rDWI and relative rADC derived from high $b$ value DWI and their diagnostic values in evaluating the onset time of acute ischemic stroke remains unclear (15-17).

The aim of the present study was to evaluate the temporal evolution of rDWI and rADC in three different $b$ values, and further compare their diagnostic performances in identifying the onset time of acute ischemic stroke, based on an embolic canine model with clear onset time $(18,19)$.

\section{Materials and methods}

Animal preparation and model establishment. All animal care and experimental procedures were performed in accordance 
with regulations specified by the Guide for the Care and Use of Laboratory Animals (NIH Publication No. 85-23, revised 1996) and the protocol was approved by the Institutional Animal Care and Use Committee of Nanjing Medical University.

A total of 12 male beagles (13-15 kg; Laboratory Animal Center of Nanjing Medical University) were anesthetized by intravenous injection with $3 \mathrm{ml} / \mathrm{kg}$ pentobarbital (Pentobarbital Sodium Salt; Chemical Reagent Company, Shanghai, China). Detailed procedures were described in our previous studies (18-20). In brief, two autologous clots (1.4 and $1.7 \mathrm{~mm}$ in diameter and $5 \mathrm{~mm}$ in length) were injected into the left middle cerebral artery (MCA). Then, after the occlusion of MCA was confirmed by angiography (Axiom Artis; Siemens AG, München, Germany), a 5-F catheter (Terumo Medical Corporation, Tokyo, Japan) was guided $2 \mathrm{~cm}$ distally to the orifice of the ipsilateral internal cerebral artery (ICA) to block the blood flow for $2 \mathrm{~h}$. After successful embolization, animals were transported to the MR imaging suite for MR scanning.

$M R$ imaging. MR scanning was performed in a 3.0-T MR whole-body scanner (Magnetom Trio; Siemens Medical Solutions, Erlangen, Germany). An 8-channel, transmit-receive extremity coil $(15 \mathrm{~cm}$ in diameter) was used. Different b values DWI acquisitions were performed at a serial of time points of 1,2,3, 4, 5 and $6 \mathrm{~h}$ after MCAO, respectively. DWI scanning parameters were as following: Twenty-two coronal sections acquired covered the entire brain; b values were 50/1,000,50/2,000 and 50/3000, with $\mathrm{TR} / \mathrm{TE}=5,500 / 97 \mathrm{msec}, 5,500 / 105 \mathrm{msec}$ and $5,500 / 110 \mathrm{msec}$, respectively; field-of-view $=200 \mathrm{~mm}$, matrix $=192 \times 192$, section thickness/gap $=2 / 0 \mathrm{~mm}, \mathrm{NEX}=4$. In addition, other MRI sequences, such as T2, FLAIR, TOF and SWI, were acquired in the same session. Each acquisition required $\sim 40 \mathrm{~min}$. All ADC maps were generated from 2-point analysis on a pixel basis. ADC values were calculated as follows:

$$
\mathrm{ADC}=\ln \left(\mathrm{SI}_{0} / \mathrm{SI}_{1}\right) /\left(\mathrm{b}_{1}-\mathrm{b}_{0}\right)
$$

in which $\mathrm{SI}_{0}$ and $\mathrm{SI}_{1}$ are the signals at $\mathrm{b}=50$ and $\mathrm{b}=1,000,2,000$ and 3,000 respectively. All the dogs were transferred back to the animal facility after MR scanning.

Imaging assessment. Hyper-intensity on DWI $(b=3,000)$ along with hypo-intensity on ADC map were defined as hyper-acute infarction. Circular regions of interest (ROI) on MR images were measured by two neuroradiologists. Hyper-acute infarctions were detected in all dogs on the DWI images of high $b$ value $(b=3,000)$ within $1 \mathrm{~h}$ after MCAO, so ROI was positioned on initial DWI $(b=3,000)$ images $(1 \mathrm{~h})$ that showed the most obvious lesions, and the corresponding mirror ROI was placed at the same position on contralateral cerebral hemisphere. After the ROI was determined on the initial DWI images, the same ROIs were placed at the same location on the DWI images with other $b$ values. Average ROI size was $150.4 \pm 37.2 \mathrm{~mm}^{2}$.

The rDWI and rADC were calculated using the following equations, respectively:

\author{
$\mathrm{rDWI}=\mathrm{SI}_{\mathrm{ischemic}} / \mathrm{SI}_{\text {normal }}$ \\ $\mathrm{rADC}=\mathrm{ADC}_{\text {ischemic }} / \mathrm{ADC}_{\text {normal }}$.
}

Statistical analysis. Repeated-measure analysis was used to assess the longitudinal changes of mean rDWI and rADC of three $b$ values at six different time points. Receiver operating characteristic (ROC) curve was used to analyze the diagnostic performance of rDWI and rADC value for identifying the ischemic lesion within $3 \mathrm{~h}$ (including $3 \mathrm{~h}$ ). Next, ROC curves for rDWI and rADC of three $b$ values in identifying hyper-acute lesions within $3 \mathrm{~h}$ were paired and compared using Wilcoxon analysis. The linear regression curve was plotted using GraphPad Prism statistical analysis software (version 5.0; GraphPad Software, Inc., San Diego, CA, USA). All numerical data are reported as the mean \pm standard deviation. SPSS software, version 17.0 (SPSS, Inc., Chicago, IL, USA) was used for all statistical analysis. $\mathrm{P}<0.05$ was considered statistically significant.

\section{Results}

Ischemic model establishment and lesion detection. A total of 12 stroke models were successfully established without any complication. All lesions could be detected using high $\mathrm{b}$ values $(\mathrm{b}=2,000$ and 3,000) DWI images since $1 \mathrm{~h}$ after MCAO. However, the ischemic lesions of only 11 dogs could be observed on standard $b$ value $(b=1,000)$ DWI images, leaving one appeared normal on standard $b$ value $(b=1,000)$ DWI image at $1 \mathrm{~h}$ after MCAO. All ADC maps appeared hypointensive in all models at all the time points. Mean rDWI and $\mathrm{rADC}$ values of three $\mathrm{b}$ values at different time points after MCAO are shown in Table I. At each time point the DWI signal intensity of the ischemic region is significantly higher compared with the contralateral normal region, while the ADC value of the ischemic region is significantly lower than the contralateral normal value $(\mathrm{P}<0.05)$. The temporal changes of ischemic lesion on DWI and ADC maps after MCAO at three $b$ values are shown in Figs. 1 and 2.

Association between $r D W I$ and $r A D C$ over time after MCAO. The rSIs of DWI images with three different $b$ values $(1,000$, 2,000 and 3,000 ) were all linearly correlated to time within $6 \mathrm{~h}$ after MCAO $(r=+0.73,+0.77$ and +0.81 , respectively; $\mathrm{P}<0.05)$. However rADCs appeared poor correlation to time at $b$ values of 1,000 and 3000 ( $\mathrm{r}=-0.29$ and -0.26 , respectively; $\mathrm{P}<0.05$ ). When $b$ value is set to 2,000 , there was no significant correlation with time ( $\mathrm{P}=0.074)$. The rSIs of DWI images with three different $b$ values increased continuously and significantly until $4 \mathrm{~h}$ after stroke $(\mathrm{P}<0.05)$, compared with each previous time point. The rADCs rapidly and significantly decreased within $1 \mathrm{~h}$ after stroke, then were relatively stable. No significant differences were detected at all the time points at $b$ values of 2,000 and 3,000 (both $P>0.05$ ), while at $a b$ value of 1,000 there was statistical difference between 1 and $2 \mathrm{~h}(\mathrm{P}<0.05)$. The changes in rDWI and rADC over time are shown in Fig. 3.

Association between $r D W I$ and $r A D C$ at different $b$ values. All rDWI values appeared to be higher at a high $b$ value compared with a standard $b$ value $(b=1,000$ and 3,000 ; $\mathrm{P}<0.05)$. There were no significant differences at 1,5 and $6 \mathrm{~h}$ 
Table I. Mean values of rDWI and rADC of three $b$ values at different time points.

\begin{tabular}{|c|c|c|c|c|c|c|}
\hline \multirow[b]{2}{*}{ Time (h) } & \multicolumn{3}{|c|}{ rDWI (b value) } & \multicolumn{3}{|c|}{ rADC (b value) } \\
\hline & 1,000 & 2,000 & 3,000 & 1,000 & 2,000 & 3,000 \\
\hline 1 & $1.43 \pm 0.22$ & $1.48 \pm 0.19$ & $1.59 \pm 0.15^{\mathrm{a}}$ & $0.57 \pm 0.05$ & $0.63 \pm 0.06^{c}$ & $0.66 \pm 0.06^{\mathrm{c}}$ \\
\hline 2 & $1.60 \pm 0.18$ & $1.78 \pm 0.14^{\mathrm{a}}$ & $1.84 \pm 0.15^{\mathrm{a}}$ & $0.57 \pm 0.04$ & $0.62 \pm 0.06^{\mathrm{c}}$ & $0.64 \pm 0.07^{\mathrm{c}}$ \\
\hline 3 & $1.77 \pm 0.21$ & $1.94 \pm 0.18^{\mathrm{a}}$ & $2.11 \pm 0.16^{\mathrm{a}, \mathrm{b}}$ & $0.56 \pm 0.04$ & $0.60 \pm 0.05^{\mathrm{c}}$ & $0.63 \pm 0.06^{c}$ \\
\hline 4 & $1.92 \pm 0.25$ & $2.18 \pm 0.31^{\mathrm{a}}$ & $2.31 \pm 0.26^{\mathrm{a}}$ & $0.54 \pm 0.03$ & $0.60 \pm 0.05^{\mathrm{c}}$ & $0.62 \pm 0.06^{c}$ \\
\hline 5 & $2.08 \pm 0.27$ & $2.27 \pm 0.31$ & $2.40 \pm 0.24^{\mathrm{a}}$ & $0.55 \pm 0.05$ & $0.59 \pm 0.05^{\mathrm{c}}$ & $0.63 \pm 0.04^{c, c}$ \\
\hline 6 & $2.13 \pm 0.26$ & $2.33 \pm 0.22$ & $2.50 \pm 0.30^{\mathrm{a}}$ & $0.53 \pm 0.04$ & $0.59 \pm 0.08$ & $0.59 \pm 0.08$ \\
\hline
\end{tabular}

${ }^{\mathrm{a}} \mathrm{P}<0.05$ vs. rDWI $(\mathrm{b}=1000)$; ${ }^{\mathrm{b}} \mathrm{P}<0.05$ vs. rDWI $(\mathrm{b}=2000) ;{ }^{\mathrm{c}} \mathrm{P}<0.05$ vs. $\mathrm{rADC}(\mathrm{b}=1000)$; ${ }^{\mathrm{d}} \mathrm{P}<0.05$ vs. rADC $(\mathrm{b}=2000)$. rDWI, relative diffusion weighted image; rADC, relative apparent diffusion coefficient.

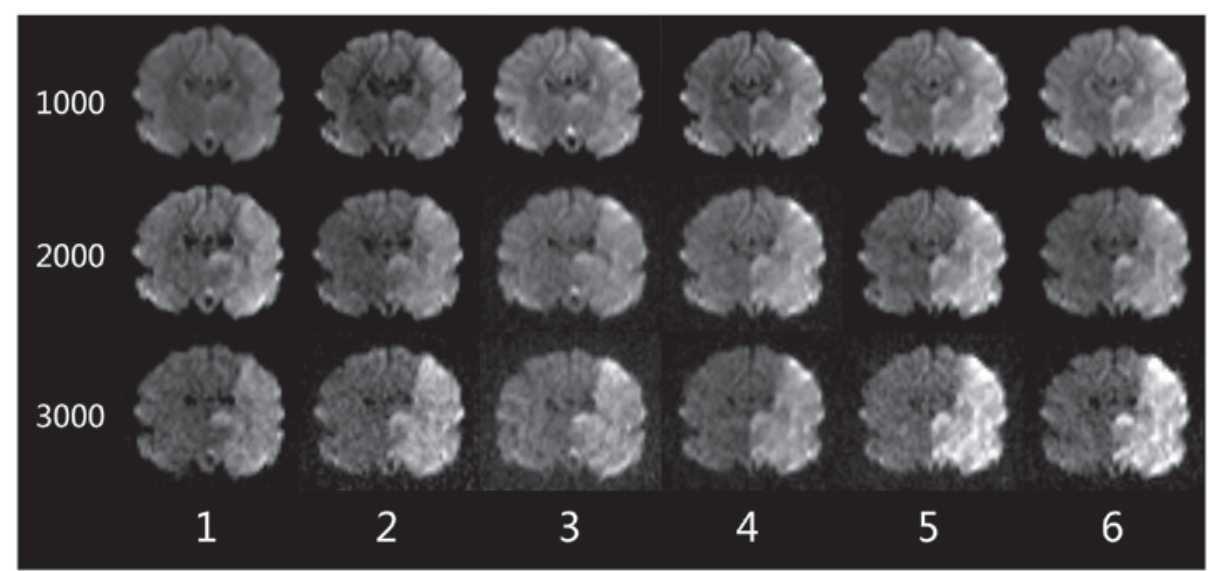

Figure 1. Longitudinal change in hyperacute ischemic lesions on diffusion weighted image (DWI) images with three different $b$ values ( $b=1,000,2,000$ and $3,000)$ at each time point after middle cerebral artery occlusion $(\mathrm{MCAO})$ in a canine model. Hyperintensive lesion can be detected on high $\mathrm{b}$ values $(\mathrm{b}=2,000$ and 3,000) DWI images since $1 \mathrm{~h}$ after MCAO. On standard $\mathrm{b}$ value $(\mathrm{b}=1,000)$ DWI image at the $1 \mathrm{~h}$ time point the same region appeared normal.

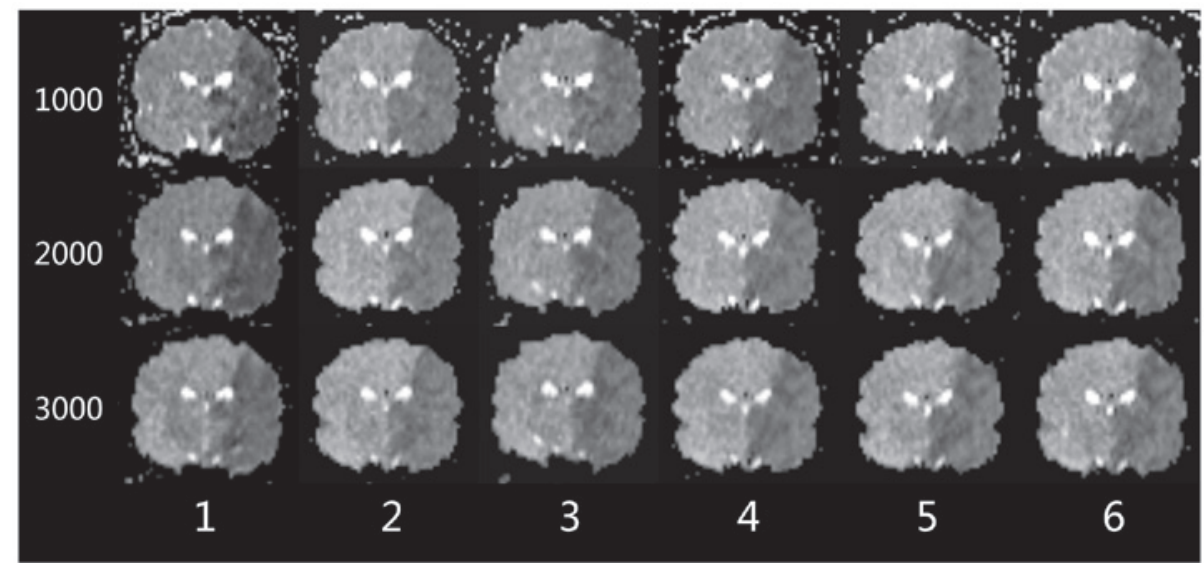

Figure 2. Longitudinal change in hyperacute ischemic lesions on apparent diffusion imaging maps with three different $b$ values $(b=1,000,2,000$ and 3,000$)$ at each time point after middle cerebral artery occlusion in a canine model.

time points between $\mathrm{b}$ values of 1,000 and 2,000, whereas significant differences were observed at 2, 3 and $4 \mathrm{~h}$. Comparing the rDWIs at $b=2,000$ with $b=3,000)$, significant differences were detected at $3 \mathrm{~h}$ after MCAO, but not at 1, 2, 4,5 and $6 \mathrm{~h}$ after.
At every time point except $6 \mathrm{~h}(\mathrm{P}=0.064)$ after $\mathrm{MCAO}$, the rADCs appeared higher at high $b$ value compared with the standard $b$ value $(b=1,000$ and $3,000, P<0.05)$. There were no significant differences at $6 \mathrm{~h}$ time points between $\mathrm{b}$ values of 1,000 and 2,000, whereas significant differences were found 


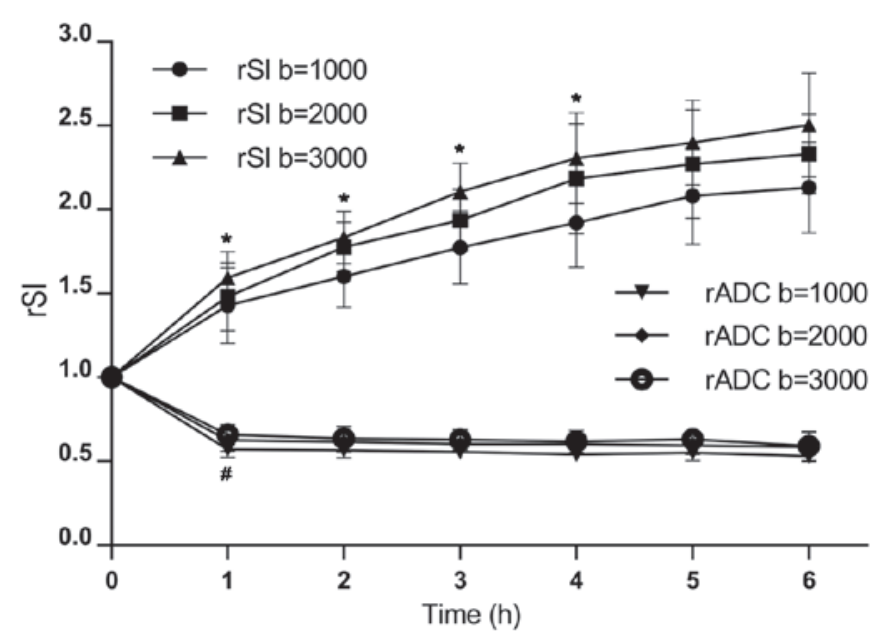

Figure 3. Time courses of relative diffusion weighted image (rDWI) and rADC after middle cerebral artery occlusion. The rDWIs with three different $b$ values increased continuously and significantly until $4 \mathrm{~h}(\mathrm{P}<0.05)$, compared with each previous time point. The rADCs rapidly and significantly decreased within $1 \mathrm{~h}$, then stabilized. ${ }^{*} \mathrm{P}<0.05$ vs. rDWI at the prior time point; ${ }^{~} \mathrm{P}<0.05$ vs. $\mathrm{rADC}$ at the prior time point. rDWI, relative signal intensity of ischemic lesions on diffusion weighted image; rSI, relative signal intensity; rADC, relative apparent diffusion coefficient.

at 1, 2, 3, 4 and $5 \mathrm{~h}$. Comparing the rDWIs at $\mathrm{b}=2,000$ and $\mathrm{b}=3,000$, significant differences were detected at $5 \mathrm{~h}$ after MCAO, but not at 1, 2, 3, 4 and $6 \mathrm{~h}$ after.

Comparison of the diagnostic performances within $3 \mathrm{~h}$ between $r D W I$ and rADC of different $b$ values. The AUC of the ROC for $\mathrm{rADC}$ at different $\mathrm{b}$ values in identifying ischemic lesions within $3 \mathrm{~h}$ were 0.645 ( $b=1,000,95 \%$ CI: $0.523-0.754$ ), $0.583(b=2,000,95 \%$ CI: $0.460-0.698)$ and $0.599(b=3,000$, 95\% CI: 0.477-0.713)

The AUC values of the ROCs for rDWI at different $\mathrm{b}$ values in identifying ischemic lesions within $3 \mathrm{~h}$ were 0.897 ( $b=1,000,95 \%$ CI: 0.803-0.956), 0.929 ( $b=2000,95 \%$ CI: $0.843-0.976)$ and 0.938 (b=3,000, 95\% CI: 0.855-0.981). The optimal threshold values for rDWI at different $b$ values were 1.67 (sensitivity, 0.972; specificity, 0.667), 1.94 (sensitivity, 0.917; specificity 0.806) and 2.18 (sensitivity, 0.889; specificity, 0.889). Among rDWIs at different $b$ values respectively, a higher $\mathrm{b}$ value indicated larger AUC, although the differences did not reach significant $(\mathrm{P}>0.05)$ (Figs. 4 and 5).

\section{Discussion}

The results of the present study suggest that rDWI at all three tested $b$ values appeared to be a useful parameter for predicting the onset time of ischemic stroke. Furthermore, the high $\mathrm{b}$ value DWI had a higher detection rate for ischemic lesions compared with the standard b value DWI. High $\mathrm{b}$ value DWI was able to detect the hyper-acute ischemic lesions which appear normal on standard b value DWI. Furthermore, rDWI at high $\mathrm{b}$ value seemed to be a better parameter than $\mathrm{rDWI}$ at standard $\mathrm{b}$ value in predicting ischemic stroke, although the differences were not significant. Secondly, relative lower AUC values were acquired when using rADC to predict whether the stroke onset time was

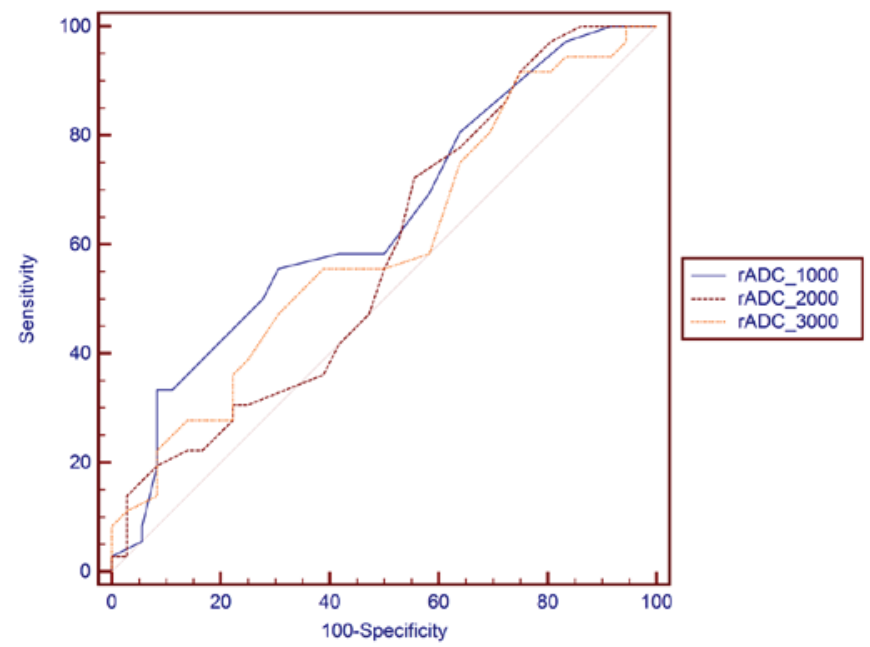

Figure 4. Receiver operating characteristic values for rADC with different $\mathrm{b}$ values in identifying ischemic lesions within $3 \mathrm{~h}$. The area under the curve values were $0.645(b=1,000$, 95\% CI: $0.523-0.754)$, $0.583(b=2,000,95 \%$ CI: $0.460-0.698)$ and $0.599(b=3,000,95 \%$ CI: 0.477-0.713). rADC, relative apparent diffusion coefficient.

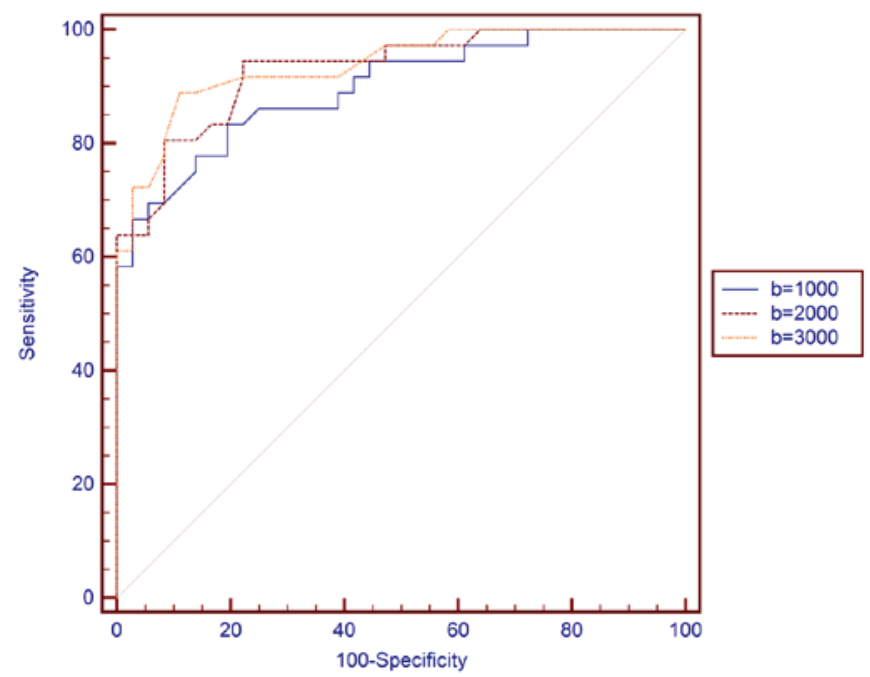

Figure 5. Receiver operating characteristic values for relative diffusion weighted imaging (rDWI) at different $b$ values in identifying ischemic lesions within 3 h. The area under the curve values were $0.897(b=1,000,95 \%$ CI: 0.803-0.956), 0.929 ( $b=2,000,95 \%$ CI: 0.843-0.976), 0.938 ( $b=3,000,95 \%$ CI: 0.855-0.981). The optimal threshold value for rDWI at different $b$ values were 1.67 (sensitivity: 0.972 , specificity: 0.667), 1.94 (sensitivity: 0.917 , specificity: 0.806) and 2.18 (sensitivity: 0.889, specificity: 0.889).

within $3 \mathrm{~h}$ or not. The rADC at all three tested $\mathrm{b}$ values may not be a suitable marker for predicting the onset time of a stroke event.

The ADC value, which was sensitive for hyper-acute ischemic stroke, was able to detect the water molecule diffusion alteration at $30 \mathrm{~min}$ after stroke onset $(4,5)$. Previous studies have demonstrated its value on depicting the pathological process of ischemic events $(21,22)$. However, at present a number of studies have proposed that there may not be a close correlation between neuronal damage and the ADC value $(23,24)$. In the present study, the AUC of the ROC values for rADC in identifying ischemic lesions within $3 \mathrm{~h}$ were $0.645,0.583$ and 0.599 at $\mathrm{b}$ values of 1,000 , 
2,000 and 3,000, respectively, suggesting that the rADC may not be a reliable predictor of onset time. Thus we insisted that ADC value was not a reliable predictor of irreversible tissue damage, particularly for ischemic events exceeding $3 \mathrm{~h}$ (25).

Compared with the rADC results, rDWI appears to be a superior marker for identifying ischemic lesions with an onset time within $3 \mathrm{~h}$. In the present study, the rDWIs of three $b$ values increased continuously and significantly until $4 \mathrm{~h}$, compared with each previous time point. The AUC of ROC values for rDWI in identifying ischemic lesions within $3 \mathrm{~h}$ were $0.897,0.929$ and 0.938 at $\mathrm{b}$ values of 1,000 , 2,000 and 3,000, respectively. These results were consistent with prior animal studies. However, a number of other studies indicated no change in rDWI within the first $24 \mathrm{~h}$, particularly in the posterior circulation and lacunar stroke. We speculated that this was due to various causes resembling ischemic stroke, and that different underlying mechanisms of stroke may have resulted in the contradictory findings. Furthermore, hypothesized that during our ischemic model establishment the cerebral flow was heavily blocked and predominantly no early recanalization was detected in the hyper-acute period, thus the ischemic damage progressed gradually after stroke onset, which was reflected in the result with the continuously increased rDWI. However, in certain prior studies, particularly for cases of lacunar stroke, the occluded vessels were recanalized prior to MRI scanning, thus the rDWI would not increase continuously. In summary, rDWI may be a useful predictor of ischemic events; however, its value should be discussed in detail after classifying the etiology of ischemic events.

The rDWIs appeared higher at high $b$ value $(b=3,000)$ compared with the standard $b$ value $(b=1,000)$ at the majority of time points, which suggests that the contrast ratio of DWI intensity at high $b$ value is higher compared with the standard $b$ value (13). The same pattern was observed with rADC values, which showed higher at high $b$ value $(b=3,000)$ compared with the standard $b$ value $(b=1,000)$. As the ADC value in ischemic tissue is lower compared with normal tissue, the contrast ratio of ADC value at high $b$ value is lower than that at standard $b$ value $(6,16,17)$. The reason for this may be the smaller degrees of ADC and DWI reduction in ischemic tissue compared with normal tissue. The exact mechanism of this difference between the normal and ischemic tissue is not yet clear. Brugieres et al (26) reported that it may be due to slow diffusing component fraction, which might be reflected more on MR imaging at higher $b$ value. Also, based on the DWI principle:

$$
\text { DWI SI }=\mathrm{SI}_{0} \times \mathrm{e}^{-\mathrm{bADC}}
$$

The DWI SI is influenced by the diffusion gradient and ADC. When the $b$ value increases, the effect of increased diffusion gradient overwhelms the decreased ADC value. Such may be reflected in the result with higher contrast ratio of DWI signal intensity, which could be regarded as the same as rDWI (6).

The diagnostic value of high $b$ value DWI in hyperacute ischemic stroke remains controversial. Purroy et al (12) and Meyer et al (16) had ever indicated that the high b value had no impact on diagnosis of acute ischemic stroke. Furthermore, the NEX parameter needed to be increased to raise the SNR which would extend the MRI scan time $(10,16,17)$. Therefore, Meyer et al suggested that the high b value DWI was not a practical stroke image modality. By contrast, a number of previous studies have speculated that the high $b$ value DWI had its distinctive value in hyper-acute ischemic stroke, for instance that high $b$ value DWI could detect the ischemic lesions which appeared as normal in standard b value DWI. Based on the present results, we would support the latter view, for three reasons: Firstly, high $b$ value $(b=3,000)$ DWI was ale to detect all ischemic lesions from $1 \mathrm{~h}$ after MCAO while standard $b$ value $(b=1,000)$ DWI missed one lesion at $1 \mathrm{~h}$ after MCAO, which was accordant to previous studies (11-13). The high b value DWI had a higher detection rate for ischemic lesions compared with standard b value DWI. Secondly, ROC results indicated that rDWI at higher b value was better than standard b value in identifying hyper-acute ischemic stroke, although differences were not significant. The statistical discrepancy might be caused by the limited size of the sample. Further studies with larger samples are required to clarify whether high $b$ value rDWI could be a better index to age the ischemic lesions. Thirdly, we did not increase the NEX so as to increase the SNR in the present study, all the DWI scans had equal scan time. In addition, the noise was acceptable. Therefore, the SNR or image quality as well as the scan time should not be a barrier to the clinical use of high $b$ value DWI in the setting of hyper-acute stroke.

There were a number of limitations to the present study. Firstly, we acquired the images at an interval of $1 \mathrm{~h}$, and the time point of $4.5 \mathrm{~h}$ for clinical thrombolysis was excluded from acquisition. Although we can explain that there were other MRI sequences, such as TOF, SWI also acquired in one session (the acquisition time was $\sim 40$ min once), the time point of $4.5 \mathrm{~h}$ should be admitted in future study. Secondly, how rADC and rDWI rapidly change within $1 \mathrm{~h}$ after MCAO remains unclear, this requires further evaluation in future studies. Thirdly, to further determine the diagnostic value of high b value DW imaging, the sample size needs to be increased in future studies.

High $b$ value DWI had a higher detection rate for hyper-acute ischemic lesion compared with standard $b$ value DWI. The rDWI may be helpful in identifying hyper-acute ischemic stroke within the first $3 \mathrm{~h}$ after stroke based on the present canine stroke model, while rADC was not. The rDWI of high $b$ value DWI seemed to provide better diagnostic value in predicting the onset time of ischemic stroke compared with standard b value DWI. Further studies with larger sample sizes are required to clarify the value of high b value rDWI.

\section{Acknowledgements}

The authors thank Dr Di Ai (Department of Anesthesia and Perioperative Medicine, University of Texas MD Anderson Cancer Center, Houston, TX, USA) for English editorial assistance. This research was funded by the National Natural Science Foundation of China (grant no. 81471764, 81401497 and 81401383) and by the National Basic Research Program of China (grant no. 2013CB733803). 


\section{References}

1. Wu CJ, Wang Q, Zhang J, Wang XN, Liu XS, Zhang YD and Shi HB: Readout-segmented echo-planar imaging in diffusion-weighted imaging of the kidney: Comparison with single-shot echo-planar imaging in image quality. Abdom Radiol (NY) 41: 100-108, 2016.

2. Xu XQ, Cheng QG, Zu QQ, Lu SS, Yu J, Sheng Y, Shi HB and Liu S: Comparative study of the relative signal intensity on DWI, FLAIR, and T2 images in identifying the onset time of stroke in an embolic canine model. Neurol Sci 35: 1059-1065, 2014.

3. Liu S, Xu X, Cheng Q, Zu Q, Lu S, Yu J, Liu X, Wang B, Teng G and Shi H: Simple quantitative measurement based on DWI to objectively judge DWI-FLAIR mismatch in a canine stroke model. Diagn Interv Radiol 21: 348-354, 2015.

4. Mohr JP, Biller J, Hilal SK, Yuh WT, Tatemichi TK, Hedges S, Tali E, Nguyen H, Mun I and Adams HP Jr: Magnetic resonance versus computed tomographic imaging in acute stroke. Stroke 26: $807-812,1995$

5. Gonzalez RG, Schaefer PW, Buonanno FS, Schwamm LH, Budzik RF, Rordorf G, Wang B, Sorensen AG and Koroshetz WJ: Diffusion-weighted MR imaging: Diagnostic accuracy in patients imaged within $6 \mathrm{~h}$ of stroke symptom onset. Radiology 210 : 155-162, 1999

6. Kim JH, Na DG, Chang KH, Song IC, Choi SH, Son KR, Kim KW and Sohn CH: Serial MR analysis of early permanent and transient ischemia in rats: Diffusion tensor imaging and high $\mathrm{b}$ value diffusion weighted imaging. Korean J Radiol 14: 307-315, 2013.

7. Xu XQ, Zu QQ, Lu SS, Cheng QG, Yu J, Sheng Y, Shi HB and Liu S: Use of FLAIR imaging to identify onset time of cerebral ischemia in a canine model. AJNR Am J Neuroradiol 35: 311-316, 2014.

8. Lansberg MG, Thijs VN, O'Brien MW, Ali JO, de Crespigny AJ, Tong DC, Moseley ME and Albers GW: Evolution of apparent diffusion coefficient, diffusion-weighted and T2-weighted signal intensity of acute stroke. AJNR Am J Neuroradiol 22: 637-644, 2001.

9. Schlaug G, Siewert B, Benfield A, Edelman RR and Warach S: Time course of the apparent diffusion coefficient (ADC) abnormality in human stroke. Neurology 49: 113-119, 1997.

10. DeLano MC, Cooper TG, Siebert JE, Potchen MJ and Kuppusamy K: High-b-value diffusion-weighted MR imaging of adult brain: Image contrast and apparent diffusion coefficien map features. AJNR Am J Neuroradiol 21: 1830-1836, 2000.

11. Lettau $\mathrm{M}$ and Laible $\mathrm{M}$ : 3-T high-b-value diffusion-weighted MR imaging in hyperacute ischemic stroke. J Neuroradiol 40: 149-157, 2013

12. Purroy F, Begue R, Quílez A, Sanahuja J and Gil MI: Contribution of high-b-value diffusion-weighted imaging in determination of brain ischemia in transient ischemic attack patients. J Neuroimaging 23: 33-38, 2013.
13. Cihangiroglu M, Citci B, Kilickesmez O, Firat Z, Karlıkaya G, Ulug AM, Bingol CA and Kovanlikaya I: The utility of high b-value DWI in evaluation of ischemic stroke at 3T. Eur J Radiol 78: 75-81, 2011.

14. Lettau $M$ and Laible M: 3-T high-b-value diffusion-weighted MR imaging of hyperacute ischemic stroke in the vertebrobasilar territory. J Neuroradiol 39: 243-253, 2012.

15. Burdette JH and Elster AD: Diffusion-weighted imaging of cerebral infarctions: Are higher B values better? J Comput Assist Tomogr 26: 622-627, 2002.

16. Meyer JR, Gutierrez A, Mock B, Hebron D, Prager JM, Gorey MT and Homer D: High-b-value diffusion-weighted MR imaging of suspected brain infarction. AJNR Am J Neuroradiol 21: $1821-1829,2000$.

17. Kim HJ, Choi CG, Lee DH, Lee JH, Kim SJ and Suh DC: High-b-value diffusion-weighted MR imaging of hyperacute ischemic stroke at 1.5T. AJNR Am J Neuroradiol 26: 208-215, 2005.

18. Zu QQ, Liu S, Xu XQ, Lu SS, Sun L and Shi HB: An endovascular canine stroke model: Middle cerebral artery occlusion with autologous clots followed by ipsilateral internal carotid artery blockade. Lab Invest 93: 760-767, 2013.

19. Liu S, Hu WX, Zu QQ, Lu SS, Xu XQ, Sun L, Zhou WZ and Shi HB: A novel embolic stroke model resembling lacunar infarction following proximal middle cerebral artery occlusion in beagle dogs. J Neurosci Methods 209: 90-96, 2012.

20. Lu SS, Liu S, Zu QQ, Xu XQ, Yu J, Wang JW, Zhang Y and Shi HB: In vivo MR imaging of intraarterially delivered magnetically labeled mesenchymal stem cells in a canine stroke model. PLoS One 8: e54963, 2013.

21. Benveniste H, Hedlund LW and Johnson GA: Mechanism of detection of acute cerebral ischemia in rats by diffusion-weighted magnetic resonance microscopy. Stroke 23: 746-754, 1992.

22. Duong TQ, Ackerman JJ, Ying HS and Neil JJ: Evaluation of extra- and intracellular apparent diffusion in normal and globally ischemic rat brain via 19F NMR. Magn Reson Med 40: 1-13, 1998.

23. Cvoro V, Marshall I, Armitage PA, Bastin ME, Carpenter T, Rivers CS, Dennis MS and Wardlaw JM: MR diffusion and perfusion parameters: Relationship to metabolites in acute ischaemic stroke. J Neurol Neurosurg Psychiatry 81: 185-191, 2010.

24. Rivers CS and Wardlaw JM: What has diffusion imaging in animals told us about diffusion imaging in patients with ischaemic stroke? Cerebrovasc Dis 19: 328-336, 2005.

25. Fiehler J, Foth M, Kucinski T, Knab R, von Bezold M, Weiller C, Zeumer H and Röther J: Severe ADC decreases do not predict irreversible tissue damage in human. Stroke 33: 79-86, 2002.

26. Brugieres P, Thomas P, Maraval A, Hosseini H, Combes C, Chafiq A, Ruel L, Breil S, Peschanski M and Gaston A: Water diffusion compartmentation at high $\mathrm{b}$ values in ischemic human brain. AJNR Am J Neuroradiol 25: 692-698, 2004. 